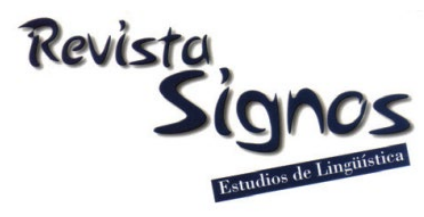

\title{
Criterios para la diccionarización de neologismos: De la teoría a la práctica ${ }^{1}$
}

\section{Criteria for the dictionarisation of neologisms: From theory to practice}

\section{Elisenda Bernal}

Profesora Agregada SERra HúNTER

UNIVERSITAT POMPEU FABRA

ESPAÑA

elisenda.bernal@upf.edu

\author{
Judit Freixa \\ UNIVERSITAT POMPEU FABRA \\ ESPAÑA \\ judit.freixa@upf.edu
}

\author{
Sergi Torner \\ UNIVERSITAT POMPEU FABRA \\ ESPAÑA \\ sergi.torner@upf.edu
}

Recibido: 06-III-2019 / Aceptado: 01-IV-2020

DOI: $10.4067 /$ S0718-09342020000300592

\section{Resumen}

La reflexión sobre los criterios para la diccionarización de los neologismos ha formado parte de la práctica lexicográfica desde sus orígenes, pero desde hace unos años existe también como línea de investigación teórica en sí misma (Ishikawa, 2006; O’Donovan \& O’Neill, 2008; Freixa, 2016; Sanmartín, 2016), gracias a lo cual se han ido identificando criterios para establecer qué unidades son más pertinentes para la actualización de los diccionarios generales. La frecuencia y la estabilidad en el uso encabezan la lista, y a veces también la cierran, sin haber tenido en cuenta otros criterios de carácter lexicográfico, pragmático, semántico o morfológico. Con el fin de refinar y acotar lo apuntado en trabajos anteriores, se presenta un análisis de neologismos frecuentes y de las últimas incorporaciones al diccionario académico que atiende los diversos factores que se establecen como criterios indicativos de la diccionarización.

Palabras Clave: Diccionario, neologismo, español, DLE, frecuencia de uso. 


\begin{abstract}
From its origins, lexicographical practice has always devoted some attention to the dictionarisation of neologisms, that is, to determining what makes a neologism adequate for inclusion in a dictionary. However, it has now become a research subfield in itself (Ishikawa, 2006; O’Donovan \& O’Neill, 2008; Freixa, 2016; Sanmartín, 2016) which has allowed for the identification of the criteria needed to establish which units are more suitable to update a general dictionary. At the top of the list, we can find frequency and stability, although these can also appear at the end of it, without having accounted for other criteria that are important from a lexicographical, pragmatic, semantic or morphological standpoint. With the aim of polishing and narrowing down what has been suggested in previous works, this paper presents an analysis of frequent neologisms and the latest additions to the Spanish academic dictionary that incorporates different factors that have been established as indicative criteria for dictionarisation.
\end{abstract}

Key Words: Dictionary, neologism, Spanish, DLE, frequency of use.

\title{
INTRODUCCIÓN
}

La reflexión sobre los criterios para la diccionarización de los neologismos ha formado parte de la práctica lexicográfica desde sus orígenes, pero desde hace unos años existe también como línea de investigación teórica en sí misma (Ishikawa, 2006; O’Donovan \& O’Neill, 2008; Freixa, 2016; Sanmartín, 2016), gracias a lo cual se han ido identificando criterios para establecer qué unidades son más pertinentes para la actualización de los diccionarios generales. En la reflexión que se lleva a cabo desde la metalexicografía, la frecuencia y la estabilidad en el uso encabezan la lista de criterios, y a veces también la cierran, sin haber tenido en cuenta otros criterios importantes de carácter lexicográfico, pragmático, semántico o morfológico. Frente a ello, la práctica lexicográfica rara vez hace explícitos los criterios que se emplean en cada obra para la inclusión de voces neológicas, más allá de afirmaciones de carácter genérico sobre la voluntad de representar el uso actual de la lengua.

Los estudios sobre neología en las lenguas naturales que se acercan al fenómeno desde la perspectiva de la confección de diccionarios suelen poner en relación, en general sin mayor discusión, la diccionariabilidad de las voces neológicas con la denominada neologicidad, esto es, el grado de novedad que supone un determinado neologismo. Según se arguye, se trata de una relación inversa: a saber, cuanto menos neológica sea una forma, mayor será su probabilidad de inclusión en los diccionarios. En términos de Sánchez Manzanares (2013: 112) "a mayor valor [de neologicidad], menor probabilidad de registro lexicográfico". Por lo que concluye (Sánchez Manzanares, 2013: 123):

"Nuestra propuesta en este trabajo consiste en la proposición del valor neológico como una variable que contribuya a la aplicación homogénea de criterios lexicográficos con respecto a la sanción de neologismos. La 
escala de neologicidad que proponemos puede constituir un punto de partida para la evaluación de otras variables que han de examinarse en la práctica lexicográfica”.

El foco de la discusión, así pues, se sitúa en un primer momento en la medición de la neologicidad de cada una de las voces candidatas a ser incorporadas en la macroestructura del diccionario, pues en función del grado de neologicidad de cada una de ellas se determinará la pertinencia de su inclusión en las obras lexicográficas. Sin embargo, hay un acuerdo unánime en la bibliografía especializada más reciente en considerar que la sanción para incluir una forma neológica en los diccionarios responde a la combinación de diferentes criterios. En este sentido, Adelstein y Freixa (2013: 3) señalan que los criterios para determinar la inclusión de voces neológicas en los diccionarios se corresponden con distintas "dimensiones del léxico (formales, semánticas, sociolingüísticas y lexicográficas), que deben ponderarse de acuerdo con el tipo de diccionario en el que se ingresarán los neologismos".

El objetivo principal de este artículo se cifra en analizar el listado de criterios propuestos por parte de la bibliografía especializada para la sanción de la neología en los diccionarios generales de lengua. Para ello, se recorre un camino de ida y vuelta desde las propuestas de la metalexicografía teórica a las respuestas que refleja la práctica lexicográfica, que en su decisión de incluir determinadas formas o excluir otras ponen de manifiesto los criterios que, aunque implícitos, han guiado la selección de voces nuevas que incrementan el lemario de los diccionarios.

Este recorrido de ida y vuelta de la teoría a la práctica se estructura en varias etapas. En un primer momento, se lleva a cabo una revisión crítica de los principales trabajos que han abordado el problema de la diccionariabilidad de voces neológicas, con el fin de obtener un estado de la cuestión actual y comprehensivo. En un segundo momento, se contrastan dichas propuestas con los criterios empleados para la inclusión de voces neológicas en los diccionarios generales de lengua, con una doble mirada. Por un lado, se examina el tratamiento que reciben en diversos diccionarios de lengua algunos neologismos de uso frecuente. Por otro, se examinan los neologismos incluidos en la última edición del DLE con el fin de determinar los criterios tenidos en cuenta por la Real Academia para la inclusión de neología; ello ha de permitir determinar si la práctica lexicográfica que refleja este diccionario puede enriquecer los criterios propuestos en la bibliografía especializada con la incorporación de nuevos criterios o la ponderación de los ya propuestos.

\section{Estado de la cuestión}

La bibliografía reciente sobre diccionarización de neología coincide en considerar que la sanción para incluir una voz neológica en los diccionarios dimana de la consideración de un conjunto de criterios de diversa índole. En concreto, los trabajos publicados en este ámbito tienen en cuenta la combinación de factores relacionados 
con la frecuencia de uso y la estabilización; con el carácter neológico, que se concreta en factores formales y semánticos; y con la sanción en otras fuentes lexicográficas de prestigio. Los apartados que siguen examinan con detalle los criterios de frecuencia, formales, semánticos y lexicográficos que se han tenido en cuenta en la bibliografía especializada para decidir sobre la diccionariabilidad de voces nuevas. La aplicación concreta de los criterios variará según las características del diccionario que se tome como referencia; los criterios deben articularse y ponderarse para cada caso concreto ${ }^{2}$.

\subsection{Criterios de frecuencia}

En general (Barnhart, 1985; Ishikawa, 2006; Cook, 2010; O’Donovan \& O’Neill, 2008; entre otros) se considera como criterio básico para el ingreso de un neologismo en los diccionarios de lengua una frecuencia de uso elevada. Así, por ejemplo, en su análisis de la neología recogida en el OED3, Ishikawa (2006: 41) concluye que "the only criteria for getting a word added is if it is being used or not. If it's out there, even if it's vulgar and non-grammatical, it will go in".

La bibliografía, sin embargo, ha discutido extensamente sobre la conveniencia de considerar la frecuencia como criterio fundamental para sancionar la diccionariabilidad de la neología. Por un lado, se ha cuestionado el papel que la frecuencia desempeña en relación con los demás criterios de diccionariabilidad. En este sentido, Freixa (2016) propone que no debe ser tomado como criterio único, sino que la frecuencia de uso es un criterio básico que actúa como primer filtro de diccionarización: todos los neologismos que ingresan en un diccionario tienen que ser frecuentes. Sin embargo, no necesariamente todos los neologismos frecuentes deben entrar en el diccionario; entre los frecuentes, tendrán entrada en los diccionarios solo aquellos neologismos que cumplan con otros criterios de diccionariabilidad.

Por otro lado, se ha puesto de relieve que la frecuencia de uso de una unidad léxica es en sí mismo un criterio complejo, pues debe dimensionarse en relación con el comportamiento temporal —estabilidad de la frecuencia sostenida en el tiempo- y de distribución de la unidad en distintos tipos de texto, registros y variedades geográficas. La idea fundamental que subyace a esta caracterización de la frecuencia es que la estabilización de una voz en el sistema de la lengua depende de su extensión en el uso en textos de tipología diversa y de modo transversal a distintas variedades, de modo que cuanto mayor sea el rango de situaciones de uso en que se emplea una voz mayor es la probabilidad de que se incorpore en la lengua, razón de su inclusión en el diccionario. En virtud de ello, se ha propuesto una conceptualización compleja de la frecuencia que atiende a cinco factores:

1) Frecuencia absoluta de uso. A mayor frecuencia de un neologismo, mayores son sus posibilidades de estabilización en la lengua y, por consiguiente, mayor es su grado 
de diccionariabilidad. En términos de Cook (2010: 35), "frequent words are more likely to be successful".

2) Extensión de uso. Los neologismos son más diccionarizables si se emplean de forma igualmente frecuente en textos de diferente tipología (Metcalf, 2002; Ishikawa, 2006). Cook (2010) matiza sin embargo que las palabras de registros no especializados tienen mayores probabilidades de estabilizarse en el uso, puesto que han pasado a formar parte de la lengua general. No obstante, Cook (2010) en su análisis de la neología estudiada por Boulanger (1997), pone en relación la frecuencia de uso con los cambios sociales que dichos neologismos reflejan, destacando que en ocasiones el uso en textos especializados tiene probabilidades mayores de estabilización:

"Interestingly, Boulanger finds that words associated with particular notional fields (e.g., disease, economics) are more likely to succeed than others. This may seem somewhat contradictory to the observation by lexicographers that occurring across a variety of genres and domains is an indication that a word is a good candidate for inclusion in a dictionary.

However, it has also been observed that the new words from a particular period of time tend to reflect what was culturally prominent then (e.g., Ayto 2006). Therefore, association with a culturally prominent notional field appears to have a positive effect on a word's success" (Cook, 2010: 35).

3) Estabilidad en el uso. El cambio léxico está sujeto a los cambios en la sociedad, tal como destaca Cook (2010: 35): "Words for referents that remained popular until the time of Boulanger's study were also found to be more likely to succeed than words whose referents were no longer popular at that time". Sin embargo, el uso de un neologismo puede ir ligado a fenómenos socioculturales pasajeros; así, neologismos con una frecuencia muy elevada en un momento dado pueden caer en desuso cuando la realidad que les ha dado carta de naturaleza desaparece. Por ello, el uso frecuente sostenido en el tiempo constituye un índice de estabilización que actúa a favor de la diccionariabilidad (Metcalf, 2002; Adelstein \& Freixa, 2013; Freixa, 2016).

4) Cambio longitudinal en la frecuencia. El uso creciente de una palabra con el paso del tiempo muestra una tendencia a su estabilización, por lo que es también un criterio de diccionariabilidad (Metcalf, 2002; Ishikawa, 2006); también lo es que el aumento en el uso no sea progresivo sino que una unidad pase de tener un carácter meramente testimonial a una elevada frecuencia de uso. El cambio longitudinal en la frecuencia indica que un vocablo existente ha pasado a ser un vocablo necesario.

5) Extensión (geográfica) en el uso. Los neologismos que se emplean en dialectos particulares difícilmente pasan a formar parte de la lengua general, por lo que se ha considerado que los utilizados en distintas variedades geolectales son mejores candidatos a ser recogidos en diccionarios de lengua (Adelstein \& Freixa, 2013). 
La frecuencia es, en definitiva, el criterio fundamental que según la bibliografía actúa como filtro de diccionariabilidad. Sin embargo, al ser las lenguas naturales objetos complejos en los que convive un gran número de variantes dialectales, diafásicas y diastráticas —es la lengua como 'complejo dialectal' (García de Diego, 1950; Borrego Nieto, 1999)_, la frecuencia de uso de una unidad léxica debe relativizarse en función de parámetros que permiten refinar las mediciones con el fin de determinar la extensión del uso de una unidad léxica; solo de este modo resulta posible obtener una imagen cabal de su estabilización en el uso, que constituye el primer filtro para determinar la incorporación de los neologismos en los diccionarios de lengua.

\subsection{Criterios formales}

Junto con la frecuencia, se han tenido en cuenta otros factores para determinar el grado de diccionariabilidad de una voz neológica, que en su conjunto examinan los procesos de formación de las palabras nuevas - ya sea desde el punto de vista semántico o meramente formal - a la luz de su conformidad con las reglas de la lengua. En este sentido, desde la perspectiva de la neologicidad se ha postulado que el carácter transgresor de un neologismo es un factor que contribuye a aumentar el sentimiento neológico — esto es, la percepción por parte de los hablantes de que se trata de una unidad neológica-, lo cual afecta a sus posibilidades de estabilización en el uso (Estopà, 2015; Bernal, 2015). Por ello, según se arguye (Freixa, 2010), la cualidad de transgresión de un neologismo es un factor que afecta inversamente a sus posibilidades de diccionarización o que incluso puede suponer su exclusión: algunos vocablos pueden forzar una regla y, con el tiempo, provocar una ampliación de la regla; en cambio, otros vocablos transgreden las reglas para obtener resultados muy llamativos, a veces agramaticales y por ende no diccionarizables.

En definitiva, pues, a mayor conformidad con las reglas de formación de la lengua, mayores las posibilidades de que la nueva unidad léxica se consolide en el uso y, por lo tanto, deba ser repertoriada en las obras lexicográficas (Sánchez Manzanares, 2013). Por ello, la bibliografía especializada ha examinado los diversos factores que intervienen en la buena formación de una unidad lingüística para determinar en qué medida influyen en su diccionarización. Los estudios analizan a este respecto los aspectos formales, objeto de atención en este epígrafe, y los aspectos semánticos, que se examinarán en el apartado siguiente.

En relación con los aspectos formales, Freixa (2016) y Adelstein y Freixa (2013) y han examinado los criterios para la inclusión de derivados neológicos en los diccionarios. Según las autoras, los diccionarios son de hecho poco sistemáticos en la inclusión de formas derivadas regulares de significado predecible. La propuesta de las autoras es que deberían incluirse siempre que sean frecuentes y presenten algún grado de impredictibilidad en alguno de los siguientes aspectos: 
a) Se produce una restricción semántica.

b) En el uso alternan diferentes variantes con diferente distribución geográfica.

c) Presentan algún tipo de impredictibilidad ortográfica.

Aunque las autoras no abundan en ello, parece evidente que la inclusión de las voces en los diccionarios cumple funciones distintas. Por un lado, informa sobre ciertas idiosincrasias semánticas del proceso derivativo, en la medida en que el significado del derivado no es meramente composicional. La propuesta es, pues, paralela al tratamiento que se defiende para la diccionarización de la derivación apreciativa (Beniers, 1990; Bernal, 2003) y de los adverbios en '-mente' (Torner, 2013). Por el otro, en cambio, el diccionario aporta información sobre el lema en cuanto signo lingüístico, informando sobre la distribución geográfica de la voz o sobre su grafía.

En relación con los derivados neológicos, Adelstein y Freixa (2013) y Freixa (2016) añaden que deben tenerse en cuenta también otras restricciones en el proceso de formación de las voces. Según ello, por lo general no son buenos candidatos a entrar en los diccionarios los derivados a partir de nombres propios, puesto que sus bases no forman parte de las nomenclaturas de los diccionarios de lengua. Asimismo, tampoco suelen incluirse los derivados formados con determinados afijos; en concreto, presentan gran resistencia a ser incorporados en los diccionarios:

a) derivados que designan corrientes ideológicas formados con '-ista', '-ismo' a partir de nombres propios y sus derivados ulteriores formados con 'anti-', 'ultra-', etc.;

b) derivados con los prefijos apreciativos 'archi-', 'hiper-' y 'mega-';

c) por norma general, derivados con 'ex-', 'vice-', 'sub-' y '-ble', a no ser que haya algún motivo específico que lo justifique.

\subsection{Criterios semánticos}

Dos son los criterios semánticos que se han tenido en cuenta para abogar por la necesidad de diccionarizar neologismos: la impredictibilidad semántica, apuntada más arriba, y la necesidad denominativa. En cuanto al primero de ellos, se acepta implícitamente el presupuesto de que cuanto menos predecible sea el significado de una unidad léxica mayor será su grado de diccionariabilidad; en cuanto al segundo, se atiende fundamentalmente a la competición de la forma neológica con una alternativa existente en la lengua.

En cuanto a la transparencia semántica, Adelstein y Freixa (2013) y Freixa (2016) y observan que el significado de un derivado es más difícil de predecir si se ha formado a partir de una base que a su vez es neológica o cuando se forma a partir de una base polisémica y toma un significado que no es el más habitual de la base. En ambos 
casos, la impredictibilidad semántica actúa como un argumento a favor de la diccionariabilidad.

Por otro lado, la impredictibilidad semántica se relaciona también con la polisemia de la forma neológica. En este sentido, el criterio fundamental que se debe tener en cuenta es la productividad polisémica: serán objeto de diccionarización los neologismos en que la polisemia regular (Apresjan, 1974) no afecte a toda la clase léxica sino solo a algunos de sus miembros (Adelstein \& Freixa, 2013). De nuevo, pues, los criterios propuestos no son exclusivos del tratamiento de la neología, sino que son coherentes con el tratamiento de la derivación en los diccionarios (Beniers, 1990).

En cuanto a la necesidad denominativa, se suele aceptar que la inexistencia de un término alternativo en la lengua para designar esa misma realidad es un hecho que favorece el éxito de un neologismo y, por ende, su estabilización, que es el paso previo necesario para su diccionarización (Sánchez Manzanares, 2013; Adelstein \& Freixa, 2013). Parece, sin embargo, que desde el punto de vista de la estabilización en el uso se trata de una cuestión fundamentalmente de corte sociolingüístico, esto es, de que exista la necesidad de la nueva voz en el modelo de comunicación en el que se incluye (Auger \& Rousseau, 1984). Por ello, la existencia de una variante alternativa puede no suponer un obstáculo sino ser un acicate en determinadas circunstancias, tal como observa Cook (2010) en su análisis del estudio de Boulanger (1997):

"Boulanger hypothesizes that in the case of competition, only the new word itself (i.e., the word form) must be accepted by speakers. In the nocompetition case, both the new word and new referent must be accepted" (Cook, 2010: 35) .

La cuestión de la necesidad denominativa se ha atendido especialmente en relación con los préstamos. Según se aduce en Adelstein y Freixa (2013) los préstamos no se incorporan a los diccionarios si existe una variante formal más adaptada, a no ser que el criterio de frecuencia aconseje lo contrario.

\subsection{Criterios documentales}

La bibliografía especializada, tal y como ha venido haciendo la práctica lexicográfica, ha examinado también como criterio para medir la diccionariabilidad la inclusión de la vOz en otros diccionarios de prestigio. En concreto, Freixa (2016) aboga por considerar como factor a favor de la repertoriación lexicográfica de los neologismos su inclusión en otras fuentes lexicográficas de prestigio de otras lenguas, mientras que en el caso del español Adelstein y Freixa (2013) aducen que debe tenerse en cuenta la inclusión de la voz en diccionarios de americanismos, ya sean integrales o diferenciales. 


\section{Del dicho al hecho}

Tal como se avanzaba al principio del artículo, nuestro objetivo es contrastar los criterios que dimanan de la discusión teórica que se ha llevado a cabo en la bibliografía especializada más reciente con el tratamiento de la neología que se observa en la práctica lexicográfica. Debe destacarse, no obstante, que, a pesar de los avances que se han producido en la reflexión lexicográfica en últimos años, la praxis de la elaboración de diccionarios no siempre muestra criterios explícitos en la selección de neología que se incorpora al lemario. Los problemas se ponen de manifiesto tanto en la no inclusión en obras de referencia de neologismos frecuentes y muy estabilizados en el uso como en la inclusión de neologismos con un uso muy restringido o que no cumplen con muchos de los criterios de diccionariabilidad propuestos en la bibliografía especializada.

En el análisis del tratamiento de la neología en los diccionarios, vamos a adoptar una perspectiva doble. Por un lado, vamos a recorrer el camino que lleva de los datos lingüísticos a los diccionarios, analizando el tratamiento que recibe en distintos diccionarios generales del español una lista de neologismos frecuentes en los corpus de lengua. Por el otro, vamos a recorrer el camino que lleva de los diccionarios a los criterios de selección de voces neológicas, analizando la neología que se ha incorporado en la última edición del DLE con el fin de contrastar las decisiones que han tomado los lexicógrafos académicos con los criterios propuestos por la bibliografía especializada.

\subsection{De los corpus a los diccionarios}

Gran parte de las críticas que reciben los diccionarios en relación con la inclusión de voces neológicas se concentra en los silencios, a veces notables, que se aprecian en la inclusión de neologismos que, desde puntos de vista diversos, parecen buenos candidatos a entrar en los diccionarios pero que, sin embargo, no han sido recogidos en distintos diccionarios del español.

En este apartado presentamos una muestra del tratamiento que reciben en varios diccionarios del español algunas voces frecuentes. Concretamente, tomamos los 50 neologismos más frecuentes recogidos por el Observatori de Neologia de la Universitat Pompeu Fabra de Barcelona <https://www.upf.edu/web/obneo> durante el período 2012-2017. Debe tenerse en cuenta que en el proyecto del Observatori se consideran neológicas las palabras que se documentan en el uso y que no aparecen en los diccionarios de referencia (Diccionario de la lengua española, DLE, y Diccionario de uso del español de América y España, DUEAE). El criterio de definición de la neología es, pues, lexicográfico: se documentan aquellas voces que no aparecen repertoriadas en el corpus lexicográfico de exclusión. Para ello se sigue una 
metodología que combina la detección semiautomática de neología con un vaciado manual de un corpus constituido por fuentes fundamentalmente de prensa general, así como también fuentes orales (Observatori de Neologia, 2004).

En el período 2012-2017 se han documentado en la base de datos del Observatori (bobneo) 14.577 neologismos en español. Entre ellos, nos centramos en las 50 unidades con una frecuencia de aparición mayor. Se trata de las unidades siguientes: app, biopic, blog, bullying, celebrity, 'chavismo', 'chavista', 'copago', 'desarrollador, -ra', 'distrital', doodle, drone/'dron', 'eurozona', fracking, hashtag, indie, 'kirchnerismo', low cost, mall, malware, 'mediocentro', 'megacomisión', 'megapíxel', 'megaproyecto', 'microtráfico', 'narcoindulto', on line/ online, playoff, 'posconflicto', post, president, reality, 'recapitalización', 'red social', 'reinventarse', retail, selfie, smartphone, 'soberanismo', 'soberanista', 'sobrecoste', spa, 'superhéroe', tablet/'tableta', trending topic, 'tuit', 'uribismo', vintage, 'yihadista' y zike.

En negrita se indican las unidades que en la actualidad ya aparecen consignadas en el diccionario académico, de manera que el primer resultado de nuestro análisis es que 11 de las 50 unidades más frecuentes ya no son neológicas desde el punto de vista lexicográfico tenido en cuenta en el proyecto del Observatori. Sin embargo, las incluimos en nuestro estudio porque dichas voces no estaban recogidas en el diccionario académico en algún momento del período y por lo tanto debían ser consideradas neológicas en ese momento.

Salvo unas pocas excepciones, se trata de neologismos muy frecuentes no únicamente en el corpus del Observatori sino también en otros corpus de referencia del español. A continuación ofrecemos el número de ocurrencias y documentos de estas voces neológicas en el corpes $\mathrm{xxi}^{3}$, ordenadas de menor a mayor frecuencia: ${ }^{4}$

\begin{tabular}{|l|l|l|}
\hline 'narcoindulto' $(1 / 1)$ & 'sobrecoste' $(120 / 84)$ & indie $(364 / 261)$ \\
'megacomisión' $(3 / 2)$ & 'mediocentro' $(140 / 116)$ & low cost $(377 / 80)$ \\
'microtráfico' $(37 / 30)$ & 'bashtag $(163 / 107)$ & 'tuit' $(390 / 202)$ \\
'soberanismo' $(47 / 28)$ & president $(265 / 174)$ & 'ika $(392 / 82)$ \\
fracking $(58 / 15)$ & malware $(270 / 107)$ & 'chavismontarse' $(411 / 356)$ \\
'posconflicto' (59/33) & 'soberanista' $(272 / 177)$ & 'megapíxel' $(560 / 328)$ \\
celebrity $(66 / 63)$ & bullying $(292 / 123)$ & mall $(605 / 393)$ \\
trending topic $(76 / 67)$ & retail $(298 / 172)$ & 'chavista' $(617 / 413)$ \\
selfie $(95 / 65)$ & 'megaproyecto' $(304 / 180)$ & 'eurozona' $(660 / 354)$ \\
'uribismo' $(95 / 54)$ & 'dron' $($ drone $)(330 / 124)$ & 'kirchnerismo' $(724 / 366)$ \\
'recapitalización' $(101 / 72)$ & vintage (330/225) & 'superhéroe' $(835 / 476)$ \\
biopic $(102 / 88)$ & 'yihadista' $(337 / 156)$ & app $(865 / 413)$ \\
doodle $(114 / 46)$ & 'copago' $(360 / 170)$ & \\
\hline
\end{tabular}

Observamos que solo 4 de los neologismos de la lista tienen una frecuencia de aparición inferior a 50 ocurrencias, mientras que 32 tienen una frecuencia superior a 200 ocurrencias y, de ellos, 17 tienen una frecuencia superior a las 500 apariciones. Desde el punto de vista de la frecuencia, pues, la gran mayoría de estas voces son 
claramente diccionarizables. Sin embargo, cumplen de modo irregular con los otros criterios establecidos por la bibliografía especializada. Desde el punto de vista formal, por ejemplo, se observa que entre los neologismos más frecuentes se encuentran unidades con características muy distintas, que ejemplifican la mayoría de los recursos de formación de palabras (prefijación, sufijación, composición propia, composición culta, etc.), si bien el peso de los préstamos crudos del inglés es muy elevado, ya que supera la mitad (26/50). Este es, sin duda, un dato que se debe tener en cuenta para matizar el criterio de la frecuencia: según la función descriptiva o prescriptiva que cumpla un diccionario, los préstamos podrán tener una presencia mayor o menor en él.

Emprendemos el análisis del tratamiento de estas voces en los diccionarios generales del español con las siguientes preguntas: ¿cómo son los neologismos más frecuentes en el uso?; ¿qué criterios de diccionarización cumplen o no cumplen dichos neologismos?; ¿qué presencia tienen en los principales diccionarios del español?

Para llevar a cabo el análisis vamos a aplicar a nuestra lista de neologismos frecuentes los criterios de diccionarización presentados en el estado de la cuestión (\$ 2); allí hemos enumerado todos los criterios documentados en la bibliografía agrupados en cuatro bloques: de frecuencia, formales, semánticos y documentales. Para esta investigación hemos tenido en cuenta solamente los criterios más representativos de cada bloque. En concreto, proponemos un baremo que nos ha de permitir medir el grado en el que cada unidad cumple con los criterios establecidos en la bibliografía, en una escala situada entre 0 y 1 para cada uno de los bloques. Los criterios aplicados y la forma de aplicación de cada uno de ellos son los siguientes:

a) Criterios de frecuencia: por tratarse de los neologismos más frecuentes del Observatori de Neologia se ha considerado que todos los neologismos tienen frecuencia alta (puntuación de 0,5) y se ha matizado el resultado teniendo en cuenta el número de ocurrencias en el corpes xxi, sumando 0,5 puntos si es igual o superior a 200 ocurrencias.

b) Criterios formales: los préstamos crudos han recibido 0 puntos, los préstamos adaptados o que no necesitan adaptación han recibido 0,5 puntos y los neologismos formados correctamente según las reglas de formación de palabras propias del español han recibido 1 punto.

c) Criterios semánticos: los neologismos denominativos han recibido 1 punto, los préstamos para los que ya existe una voz española alternativa no se han considerado denominativos y han recibido un 0 . Los préstamos denominativos han recibido 0,5 puntos, al igual que los sufijados sobre nombres propios de políticos contemporáneos.

d) Criterios documentales: se ha tenido en cuenta la inclusión de las voces en diccionarios generales de otras lenguas - GDLC, LGR, LZ y OED_- y en diccionarios del español —DEA, DLE y DUE—. Según su aparición en 
diccionarios generales de otras lenguas, han obtenido 1 punto los que aparecen en los tres diccionarios, 0,5 puntos si aparecen en dos de ellos y 0 si aparecen solamente en uno o en ninguno; de igual modo, han recibido 1 punto los neologismos que aparecen en los tres diccionarios españoles consultados, 0,5 puntos aquellos que aparecen en dos y 0 puntos si aparecen en uno o en ninguno.

En la Tabla 1 puede verse el resultado de la aplicación y cuantificación de los criterios empleados para los 50 neologismos, que aparecen ordenados según el número de criterios que cumplen (siendo 5 el máximo posible y 0,5 el mínimo): 
Tabla 1. Criterios de diccionarización aplicados a los 50 neologismos más frecuentes del período 2012-2017.

\begin{tabular}{|c|c|c|c|c|c|c|}
\hline & Frecuencia & Formales & \begin{tabular}{|l|} 
Semánticos \\
\end{tabular} & $\begin{array}{l}\text { Documentales } \\
\text { (otras lenguas) }\end{array}$ & $\begin{array}{c}\text { Documentales } \\
\text { (español) }\end{array}$ & Total \\
\hline doodle & 0,5 & 0 & 0 & 0,5 & 0 & 1 \\
\hline fracking & 0,5 & 0 & 0 & 0,5 & 0 & 1 \\
\hline mall & 1 & 0 & 0 & 0 & 0 & 1 \\
\hline malware & 1 & 0 & 0 & 0 & 0 & 1 \\
\hline president & 1 & 0 & 0 & 0 & 0 & 1 \\
\hline trending topic & 0,5 & 0 & 0 & 0 & 0,5 & 1 \\
\hline$a p p$ & 1 & 0 & 0 & 0,5 & 0 & 1,5 \\
\hline bullying & 1 & 0 & 0 & 0 & 0,5 & 1,5 \\
\hline retail & 1 & 0 & 0 & 0,5 & 0 & 1,5 \\
\hline selfie & 0,5 & 0 & 0 & 1 & 0 & 1,5 \\
\hline 'uribismo' & 0,5 & 1 & 0,5 & 0 & 0 & 1,5 \\
\hline celebrity & 0,5 & 0 & 0 & 1 & 0,5 & 2 \\
\hline hashtag & 0,5 & 0 & 0 & 1 & 0,5 & 2 \\
\hline indie & 1 & 0 & 0 & 0,5 & 0,5 & 2 \\
\hline playoff & 1 & 0 & 0 & 0,5 & 0,5 & 2 \\
\hline smartphone & 1 & 0 & 0 & 0,5 & 0,5 & 2 \\
\hline zika & 1 & 0 & 0,5 & 0,5 & 0 & 2 \\
\hline biopic & 0,5 & 0,5 & 0,5 & 1 & 0,5 & 2,5 \\
\hline 'chavismo' & 1 & 1 & 0,5 & 0 & 0 & 2,5 \\
\hline 'chavista' & 1 & 1 & 0,5 & 0 & 0 & 2,5 \\
\hline 'kirchnerismo' & 1 & 1 & 0,5 & 0 & 0 & 2,5 \\
\hline low cost & 1 & 0 & 0 & 1 & 0,5 & 2,5 \\
\hline 'megacomisión' & 0,5 & 1 & 1 & 0 & 0 & 2,5 \\
\hline 'microtráfico' & 0,5 & 1 & 1 & 0 & 0 & 2,5 \\
\hline 'narcoindulto' & 0,5 & 1 & 1 & 0 & 0 & 2,5 \\
\hline on line/online & 1 & 0 & 0 & 0,5 & 1 & 2,5 \\
\hline 'posconflicto' & 0,5 & 1 & 1 & 0 & 0 & 2,5 \\
\hline 'tuit' & 1 & 0,5 & 0,5 & 0,5 & 0 & 2,5 \\
\hline 'dron' (drone) & 1 & 0,5 & 0,5 & 1 & 0 & 3 \\
\hline 'distrital' & 1 & 1 & 1 & 0 & 0 & 3 \\
\hline 'mediocentro' & 1 & 1 & 1 & 0 & 0 & 3 \\
\hline post & 0,5 & 0 & 0,5 & 1 & 1 & 3 \\
\hline 'reinventarse' & 1 & 1 & 1 & 0 & 0 & 3 \\
\hline 'sobrecoste' & 0,5 & 1 & 1 & 0,5 & 0 & 3 \\
\hline 'copago' & 1 & 1 & 1 & 0 & 0,5 & 3,5 \\
\hline 'megapíxel' & 1 & 1 & 1 & 0,5 & 0 & 3,5 \\
\hline 'megaproyecto' & 1 & 1 & 1 & 0,5 & 0 & 3,5 \\
\hline reality & 1 & 0 & 0 & 1 & 1 & 3,5 \\
\hline 'recapitalización' & 0,5 & 1 & 1 & 1 & 0 & 3,5 \\
\hline spa & 1 & 0 & 0 & 1 & 1 & 3,5 \\
\hline 'tableta' (tablet) & 1 & 1 & 0,5 & 0 & 1 & 3,5 \\
\hline vintage & 1 & 0 & 0 & 1 & 1 & 3,5 \\
\hline blog & 1 & 0,5 & 0,5 & 1 & 1 & 4 \\
\hline 'desarrollador, -ra' & 1 & 1 & 1 & 0,5 & 0,5 & 4 \\
\hline 'red social' & 1 & 1 & 1 & 0,5 & 0,5 & 4 \\
\hline 'superhéroe' & 1 & 1 & 1 & 1 & 0 & 4 \\
\hline 'eurozona' & 1 & 1 & 1 & 0,5 & 1 & 4,5 \\
\hline 'soberanismo' & 0,5 & 1 & 1 & 1 & 1 & 4,5 \\
\hline 'soberanista' & 1 & 1 & 1 & 1 & 1 & 5 \\
\hline 'yihadista' & 1 & 1 & 1 & 1 & 1 & 5 \\
\hline
\end{tabular}


Los datos de la Tabla 1 ponen de manifiesto que entre los neologismos más frecuentes se hallan unidades con características distintas en lo relativo a sus posibilidades de inclusión en los diccionarios. En la parte baja de la tabla se sitúan algunas voces que cumplen con todos los criterios o con una gran mayoría de ellos. Encabezan esta lista las voces 'soberanista' y 'yihadista', que cumplen todos los criterios, seguidos de 'eurozona' y 'soberanista', que obtienen una puntuación de 0,5 en uno de los criterios, y blog, 'desarrollador, -ra', 'red social' y 'superhéroe', que tienen una puntuación global de 4. En la parte superior de la tabla se sitúan los neologismos que cumplen con un número de criterios menor, entre los que destacan las voces doodle, fracking, mall, malware, president y trending topic, con una puntuación de 1, y app, bullying, retail, selfie y 'uribismo'. Salvo la última voz de la lista, formada a partir de un nombre propio, se trata en todos los casos de préstamos — todos ellos préstamos del inglés, salvo president, del catalán—. La lista continúa con 6 préstamos más del inglés que tienen una puntuación de 2 según los criterios aplicados, y con 11 palabras que obtienen una puntuación de 2,5, entre las que se cuentan 4 anglicismos más y 3 derivados de nombres propios. Una primera conclusión, pues, es que los neologismos menos diccionarizables son fundamentalmente préstamos del inglés o bien derivados de nombres de políticos contemporáneos que, aun siendo altamente frecuentes, no aparecen todavía consignados en diccionarios generales de otras lenguas o diccionarios españoles de corte descriptivo.

Un segundo dato que se desprende del análisis de la Tabla 1 es que si se prescinde de los criterios documentales, hay 11 voces que obtienen la máxima puntuación en relación con todos los demás criterios — de frecuencia, formales y semánticos-: 'distrital', 'mediocentro', 'reinventarse', 'copago', 'megapíxel', 'megaproyecto', 'desarrollador, -ra', 'red social', 'superhéroe', 'eurozona', 'soberanista' y ‘yihadista'.

Parecería lógico esperar que dichas voces hubieran sido ampliamente incluidas en los diccionarios. Sin embargo, han recibido un tratamiento poco homogéneo en las fuentes lexicográficas tanto del español como de otras lenguas. Así, 'distrital', 'mediocentro' y 'reinventarse' obtienen un 0 en las dos columnas relativas a los criterios documentales, es decir, tienen una presencia escasa o nula en las fuentes lexicográficas de referencia; 'copago', 'megapíxel', 'megaproyecto', 'desarrollador, -ra' y 'red social' no obtienen un 1 en ninguna de las dos columnas, es decir, tienen cierta presencia en las fuentes lexicográficas, pero no está generalizada; y 'superhéroe', 'eurozona', 'soberanista' y 'yihadista' tienen un 1 en al menos una de las columnas, es decir, tienen una presencia rica en los diccionarios. Esta disparidad en el tratamiento pone de manifiesto que en el proceso de toma de decisiones que ha llevado a su inclusión o no en los diccionarios se han tenido en cuenta otros factores además de los referenciados en la bibliografía. 
En este mismo sentido, conviene notar, como ya hemos mencionado antes, que algunos de los 50 neologismos más frecuentes en el período estudiado han sido incorporados ya en el diccionario académico. En la Tabla 1, dichas unidades se encuentran destacadas en negrita y, como se puede observar, se acumulan en la parte baja de la tabla. Esto significa que para el diccionario académico se han escogido mayoritariamente las unidades que más criterios cumplen o, dicho de otro modo, los criterios que propone la bibliografía se aplican también para el diccionario académico.

Llaman por el contrario la atención algunas unidades que, aun cumpliendo la mayoría de los criterios, como 'eurozona', 'desarrollador, -ra', vintage, post o 'copago', no se han incorporado en el diccionario académico, aunque es de suponer que lo harán, si siguen con una frecuencia de uso elevada y si no contravienen otros criterios internos del diccionario. Inversamente, también llaman la atención las unidades 'tuit' y 'dron', ya incluidas en el diccionario académico a pesar de no cumplir tantos criterios: es evidente que ambas palabras fueron diccionarizadas tras un período relativamente breve en el uso, pero es también evidente que en ese breve período demostraron ser ya palabras diccionarizables (muy frecuentes, altamente necesarias desde el punto de vista denominativo, internacionalizadas, fácilmente adaptables, con familia derivativa, etc.).

Vamos a centrarnos ahora en el segundo criterio documental: la presencia de los neologismos en distintos diccionarios del español. Debemos empezar destacando que 17 de los 50 neologismos más frecuentes en el período 2012-2017 no aparecen en ninguno de los diccionarios consultados: 'chavismo', 'chavista', doodle, 'kirchnerismo', 'mediocentro', 'megacomisión', 'megaproyecto', 'microtráfico', 'narcoindulto', 'posconflicto', president, 'recapitalización', 'reinventarse', selfie, 'sobrecoste', 'uribismo' y zika. Como podemos ver, en esta lista se encuentran préstamos recientes y neologismos derivados de nombres propios de políticos contemporáneos, pero también otras unidades que podrían tener un lugar en el diccionario.

En la Tabla 2 pueden verse los resultados para el 66\% de neologismos restantes, es decir, los 33 neologismos frecuentes que sí han sido repertoriados en alguno de los cuatro diccionarios consultados: 
Tabla 2. Criterio documental: presencia en diccionarios generales de referencia del español.

\begin{tabular}{|l|l|l|l|l|}
\hline & NEOMM & DUE4 & DEA & DLE \\
\hline app & $\times$ & & & \\
\hline biopic & & $\times$ & $\times$ & \\
\hline blog & $\times$ & $\times$ & $\times$ & $\times$ \\
\hline bullying & & $\times$ & $\times$ & \\
\hline celebrity & $\times$ & $\times$ & & \\
\hline 'copago' & $\times$ & & $\times$ & \\
\hline 'desarrollador, -ra' & $\times$ & $\times$ & & \\
\hline 'distrital' & & & $\times$ & \\
\hline 'dron' (drone) & $\times$ & & & $\times$ \\
\hline 'eurozona' & $\times$ & $\times$ & $\times$ & \\
\hline fracking & $\times$ & & & \\
\hline bashtag & $\times$ & $\times$ & & \\
\hline indie & $\times$ & $\times$ & & \\
\hline low cost & $\times$ & $\times$ & & \\
\hline mall & & $\times$ & & \\
\hline malware & $\times$ & & & \\
\hline 'megapíxel' & $\times$ & & & \\
\hline on line/ online & $\times$ & $\times$ & $\times$ & \\
\hline playoff & & $\times$ & $\times$ & \\
\hline post & $\times$ & $\times$ & $\times$ & \\
\hline reality & $\times$ & $\times$ & $\times$ & $\times$ \\
\hline 'red social' & $\times$ & $\times$ & $\times$ & $\times$ \\
\hline retail & $\times$ & $\times$ & & \\
\hline smartphone & $\times$ & $\times$ & $\times$ & \\
\hline 'soberanismo' & $\times$ & $\times$ & $\times$ \\
\hline 'soberanista' & $\times$ & $\times$ & $\times$ \\
\hline spa & $\times$ & & $\times$ & \\
\hline 'superhéroe' & & $\times$ & $\times$ \\
\hline 'tableta' (tablet) & $\times$ & $\times$ & \\
\hline trending topic & $\times$ & & \\
\hline 'tuit' & $\times$ & & \\
\hline vintage & $\times$ & & \\
\hline 'yihadista' & $\times$ & & \\
\hline
\end{tabular}

Como puede verse, NEOMM y DUE4 incorporan la mitad de los neologismos, con una amplia coincidencia entre ambas fuentes; en cambio, en el DEA y en el DLE se documentan muchas menos voces (16 y 11, respectivamente). Así pues, los cuatro diccionarios consultados se sitúan en diferentes puntos según su grado de incorporación de neologismos: en el extremo más abierto tenemos el NEOMM, un diccionario cuyo objetivo es explícitamente recoger neologismos y, en el otro extremo, el DLE, cuya función prescriptiva limita la incorporación de neología.

De este listado de 33 voces, solo 7 aparecen repertoriadas en las cuatro obras consultadas: blog, reality, 'soberanismo', 'soberanista', spa, 'tableta' y 'yihadista'. Todas ellas tienen puntuaciones globales altas en los criterios de diccionariabilidad según los datos recogidos en la Tabla 1, si bien destaca que reality y 'tableta' no tienen puntuaciones altas en relación con los criterios formales y semánticos. 
Un segundo grupo de neologismos contiene las voces post, vintage, online/on line $\mathrm{y}$ 'eurozona', que ya aparecen en los tres diccionarios de uso, pero no están recogidas en el DLE. Como ya hemos dicho anteriormente, serían buenos candidatos para el diccionario académico, porque cumplen además la mayoría de criterios (con la excepción de online/on line, ya normalizada como 'en línea' en dicha obra). Habrá que esperar igualmente para ver qué tratamiento recibirán en la próxima actualización del diccionario normativo otros neologismos frecuentes ya documentados al menos en dos de los tres diccionarios de uso (NEOMM, DUE4 y DEA). Probablemente no se consignarán todos los préstamos del inglés que cumplen los demás criterios (indie, celebrity, trending topic, low cost, hashtag, smartphone, biopic, playoff, bullying) pero tal vez sí los neologismos formados a partir de reglas propias del español, como 'copago' y 'desarrollador, -ra'.

\subsection{De los diccionarios a los criterios de diccionariabilidad}

Un segundo modo de aproximarse al tratamiento lexicográfico de la neología consiste en examinar los criterios que subyacen a la selección de voces neológicas incorporadas en los diccionarios. Con este fin, vamos a analizar en este apartado las palabras que se han incorporado en la última edición del DLE, con el objetivo no de criticar las incorporaciones u omisiones que puedan observarse en la obra académica, sino de tratar de inferir los criterios no explícitos que se han tenido en cuenta para llevar a cabo la selección y contrastarlos con los criterios propuestos en la bibliografía especializada, pues las decisiones de la praxis lexicográfica, creemos, pueden enriquecer o matizar la discusión de la metalexicografía teórica.

En diciembre de 2017, la RAE presentó las modificaciones (3.345) que se introdujeron en la 23.a edición de 2014, de las que destacan 62 entradas nuevas. Se trata de las voces siguientes (a las que hay que añadir un formante, 'onto-'): 'amusia', 'antagonizar', 'aporofobia', 'asana', 'ataché', 'audiolibro', 'audiología', 'audiólogo, -ga', 'autólogo, -ga', 'bicatenario, -ria', 'biocida', 'bioenergía', 'bocas', 'buenismo', 'buenista', 'calefactable', 'calefactar', 'chakra', 'chusmear', 'clicar', 'cliquear', 'cliqueo', 'compostador, -ra', 'compostar', 'compostera', 'compostero', container, 'contaje', 'continentalidad', 'contraincendios', cracker, 'cubicaje', 'cupular'1 (adj.), 'cupular'2 (v. tr.), 'deportivamente', 'desalador, -ra', 'diagramador, -ra', 'discinesia', 'dispersante', 'especismo', 'especista', fair play, 'halal', holter, 'hummus', 'imafronte', kosher, 'latino, na'2, 'minicasino', 'monocatenario, -ria', 'murano', 'niguatoso, -sa', 'notas', 'pinqui', 'porro', 'postureo', 'posverdad', sharia, 'táper', 'umma' y 'vallenato'.

$\mathrm{Si}$ atendemos a los criterios que hemos presentado en el apartado anterior, se observa que se han tenido en cuenta de manera desigual, puesto que mientras algunos se cumplen de un modo generalizado, otros no parecen haberse tomado en consideración en la selección de algunas de las voces. Así, en relación con la frecuencia de uso, puede contrastarse el uso de estas voces en los dos principales corpus del 
español — crea $^{5}$ y corpes xxi, de la Real Academia- y un corpus específico de neología - bobneo, la base de datos del Observatori de Neologia - De los resultados de las búsquedas llevadas a cabo destaca el hecho de que algunas de las palabras introducidas no tienen ninguna ocurrencia en los corpus, o bien su frecuencia es muy baja (igual o inferior a 3) en todos ellos, tal como ponen de manifiesto los datos de la Tabla 3:

Tabla 3. Palabras con frecuencia baja.

\begin{tabular}{|l|l|l|l|}
\hline & bobneo & corpes xxi & crea \\
\hline 'aporofobia' & 2 & - & - \\
\hline 'calefactable' & - & $2 / 2$ & $3 / 3$ \\
\hline 'calefactar' & 3 & - & - \\
\hline 'cliqueo' & - & - & - \\
\hline 'especismo' & 1 & $1 / 1$ & - \\
\hline 'especista' & 1 & $1 / 1$ & - \\
\hline 'imafronte' & - & $1 / 1$ & - \\
\hline 'minicasino' & 2 & - & - \\
\hline 'niguatoso, -sa' & - & - & - \\
\hline 'pinqui' & - & - & - \\
\hline
\end{tabular}

Parece desprenderse de estos datos, pues, que la frecuencia no ha sido empleada en este caso como un filtro de exclusión. Algo distintos son, en cambio, los resultados que se desprenden del análisis formal pues, en efecto, todas las palabras derivadas y compuestas son palabras bien formadas (en el sentido de que siguen las reglas de buena formación de palabras); en cuanto a los 12 préstamos incorporados, proceden mayoritariamente del inglés, al que sigue de cerca el árabe:

Tabla 4. Préstamos incorporados en el DLE.

\begin{tabular}{|l|l|l|}
\hline Lengua de origen & \multicolumn{2}{|c|}{ Casos } \\
\hline árabe & 4 & 'halal', 'hummus' (o 'humus'), sharía (o sharia), 'umma' \\
\hline francés & 1 & 'ataché' \\
\hline inglés & 5 & container, cracker, fair play, kosher, 'táper' \\
\hline sánscrito & 2 & 'asana', 'chakra' (o 'chacra') \\
\hline
\end{tabular}

Destaca asimismo positivamente la inclusión de familias de palabras, que dota de consistencia al diccionario en el sentido de que se ofrecen series derivativas completas o bien se completan series derivativas que ya contaban con algún representante en el diccionario. Así sucede con 'audiología' y 'audiólogo, -ga'; 'buenismo' y 'buenista'; 'calefactable' y 'calefactar' (que completan la serie ya presente en el diccionario de 'calefactor, -ra' y 'calefactorio'); 'clicar', 'cliquear' y 'cliqueo' (que completan la serie de 'clic'); 'compostador, -ra', 'compostar', 'compostera' y 'compostero' (que completan la serie de 'compost' y 'compostaje'), y 'especismo' y 'especista'. Una mención aparte merece el caso de 'postureo', que se ha introducido sin el verbo del cual procede, si bien se menciona explícitamente en la etimología: ${ }^{6}$ 


\section{postureo}

Del coloq. posturear 'actuar con postureo', y este de postura y -ear.

m. coloq. Esp. Actitud artificiosa e impostada que se adopta por conveniencia o presunción.

Finalmente, se incluye un adverbio en '-mente', la voz 'deportivamente' ('con deportividad'). Su inclusión puede parecer sorprendente, pues tiene un significado predictible y se emplea únicamente con el uso prototípico de adverbio de modo característico de los adverbios deajetivales en '-mente'. Sin embargo, tal vez su inclusión pueda quedar justificada debido al hecho de que se emplea con frecuencia en el sentido figurado de 'aceptando de buen grado una situación adversa', y no únicamente referido a los deportes; sin embargo, esta acepción ya está en el adjetivo de base, aunque este sentido no se indica ni en la entrada del adjetivo ni en la entrada del adverbio — aunque sí se ha definido en la entrada del sustantivo-:

\section{deportividad}

1. f. Proceder deportivo (\| ajustado a normas de corrección y respeto propias del deporte).

2. f. Esp. Actitud de quien acepta de buen grado una situación adversa. Aceptó con deportividad que eligieran a otro.

\section{deportivamente}

1. $a d v$. Con deportividad.

En tercer lugar, en cuanto a los criterios semánticos, la selección de entradas que se incorporan en la última edición del DLE parece que pone de manifiesto que las voces del listado cumplen en todas las ocasiones con lo que la bibliografía especializada suele llamar necesidad denominativa. Aunque dicho concepto sea altamente subjetivo, en este caso concreto se observa que un número importante de neologismos que tienen entrada en el diccionario son voces pertenecientes a áreas temáticas científicas tales como la medicina, la bioquímica o la arquitectura, que han perdido su valor terminológico especializado al incorporarse en la lengua general, tal como se observa en la Tabla 5, sin entrar a valorar si otras palabras deberían llevar marcas diatécnicas, como fair play (deportes) o sharía (religión), por ejemplo: 
Tabla 5. Palabras marcadas técnicamente.

\begin{tabular}{|l|c|l|}
\hline \multicolumn{1}{|c|}{ Áreas temáticas } & \multicolumn{2}{c|}{ Casos } \\
\hline arquitectura & 3 & 'cupular' (adj.), 'cupular' (v. tr.), 'imafronte' \\
\hline biología y psicología & 1 & 'bioenergía' \\
\hline bioquímica & 2 & 'bicatenario, -ria', 'monocatenario,-ria' \\
\hline bioquímica y fisiología & 1 & 'antagonizar' \\
\hline física y química & 1 & 'dispersante' \\
\hline geometría, deportes y mecánica & 1 & 'cubicaje' \\
\hline informática & 1 & cracker \\
\hline medicina & 6 & $\begin{array}{l}\text { 'amusia', 'audiología', 'audiólogo, -ga', 'autólogo, -ga', 'discinesia', } \\
\text { bolter }\end{array}$ \\
\hline química & 1 & 'biocida' \\
\hline
\end{tabular}

Destaca, asimismo, la incorporación de tres casos de recategorización de nombres propios en nombres comunes, por un proceso de metonimia (NGLE, $\mathbb{S} 12.8 \mathrm{e}$ ): holter (del nombre del biofísico N. J. Holter, que inventó el monitor que lleva su nombre), 'murano' (topónimo) y 'pinqui' o pinky (adaptación de la marca registrada Pikys ${ }^{\circledR}$, donde la $-s$ final ha sido reinterpretada como marca de plural y con una '-n-' adventicia). Si bien las dos primeras tienen una presencia estable en corpes xxi, cabe señalar la variación gráfica que presentan en el corpus, puesto que oscilan entre la mayúscula y la minúscula inicial; en este sentido, la inclusión en el diccionario sanciona definitivamente su tratamiento como nombre común y orienta, al fin, su uso. El caso de 'pinqui' es aislado, puesto que no se registran datos en las bases consultadas (bobneo, corpes xxi y crea).

En cuarto lugar, en lo que concierne a los criterios documentales, no parecen haber sido decisivos para la inclusión de ciertas voces en el DLE, puesto que algunas de ellas, además de no ser frecuentes (Tabla 6), tampoco aparecen repertoriadas en otros diccionarios, ya sean de uso o de neologismos del español, ya de lenguas próximas:

Tabla 6. Presencia documental en otros diccionarios de las palabras con frecuencia baja.

\begin{tabular}{|l|l|l|l|l|l|l|l|}
\hline & dea & neomm & due4 & gdlc & oed & lgr & lz \\
\hline 'amusia' & & & & $\times$ & $\times$ & $\times$ & \\
\hline 'aporofobia' & & & & & & & \\
\hline 'asana' & $\times$ & & $\times$ & & & & \\
\hline 'ataché' & $\times$ & & $\times$ & & & & \\
\hline 'bicatenario -ria' & & & & $\times$ & & & \\
\hline 'calefactable' & & & $\times$ & & & & \\
\hline 'calefactar' & $\times$ & & & $\times$ & & & \\
\hline 'cliqueo' & & & $\times$ & & & & \\
\hline 'continentalidad' & & & $\times$ & & $\times$ & & \\
\hline 'cupular' (v. tr.) & $\times$ & & & & & & \\
\hline 'especismo' & & & & & $\times$ & $\times$ & $\times$ \\
\hline 'especista' & & & & & $\times$ & $\times$ & $\times$ \\
\hline 'imafronte' & $\times$ & & & & & & \\
\hline 'minicasino' & & & & & & & \\
\hline 'monocatenario, -ria' & & & & $\times$ & & & \\
\hline 'niguatoso, -sa' & & & & & & & \\
\hline 'pinqui' & & & & & & & \\
\hline
\end{tabular}


Se observa en los datos de la Tabla 6, en efecto, que 'aporofobia', 'minicasino', 'niguatoso, -sa' y 'pinqui' son voces exclusivas del DLE, que no se documentan en ninguno de los diccionarios de referencia consultados. En el otro extremo se sitúan las voces 'amusia', 'especismo' y 'especista', que aparecen en tres de los cuatro diccionarios de otras lenguas, hecho que refuerza la inclusión en el diccionario académico. El resto de palabras se sitúan a medio camino, pues tienen una presencia documental menor: se documentan en uno, o a veces dos, de los diccionarios consultados - del español o de otras lenguas-. Este hecho, sumado a su baja frecuencia, no podría ser decisivo para determinar su incorporación.

Finalmente, hay que mencionar la aplicación implícita de ciertos criterios pragmáticos apenas tenidos en cuenta en la bibliografía, que inciden en la inclusión de palabras marcadas por el registro coloquial y que han hecho incorporar al diccionario dos lexicalizaciones, 'bocas' y 'notas' (a su vez, con marcas diatópicas que los adscriben a España), el ya mencionado 'postureo' y el verbo 'chusmear' (con marca diatópica que lo adscribe a Argentina, Paraguay, Uruguay y Honduras). Asimismo, se han incluido dos voces más, marcadas solo diatópicamente: 'minicasino' (España) y 'niguatoso, -sa' (Venezuela), que se deben entender como exponentes de su variedad geolectal. ${ }^{7}$

\section{Discusión de los resultados}

Cada nueva edición del diccionario académico suele generar acalorados debates que inciden sobre todo en un aspecto: la sorpresa que causa que una determinada palabra que los hablantes consideran tan normal y tan habitual, es decir, tan frecuente, no aparezca todavía recogida en él. Ciertamente, el análisis demuestra que la frecuencia es uno de los criterios que se han considerado fundamentales a la hora de decidir la inclusión en los distintos diccionarios del español. Sin embargo, el análisis llevado a cabo pone de manifiesto que, al menos en el caso del DLE, la frecuencia de uso no ha sido un criterio de peso en la toma de decisiones, sino que se combina con otros criterios, más o menos evidentes, tales como la compleción de series derivativas, la actualización de campos semánticos, la orientación del uso normativo o la representatividad geolectal. Así, por ejemplo, a pesar de que 'amusia' no es una voz frecuente, actualiza el campo de la neurología, puesto que en el diccionario ya se recogían 'agnosia', 'alalia' o 'agrafía', lo cual constituye un argumento a favor de su diccionarización; la inclusión de esta voz viene reforzada, además, por la presencia en diccionarios de otras lenguas.

De hecho, tal como ha propuesto la bibliografía especializada, la frecuencia absoluta de uso en sí misma no debería ser un criterio único si no va acompañada de otros indicadores que permitan certificar la estabilización de la voz en el uso. En este sentido, por ejemplo, la inclusión de 'posverdad' en el diccionario académico es una apuesta que parece arriesgada, dada la incorporación tan reciente de esta voz al 
español, que hace difícil prever su estabilización en la lengua: al ser muy reciente, carecemos todavía de perspectiva histórica suficiente para certificar su consolidación en el uso. En cambio, un caso distinto parece ser el de 'aporofobia', que, pese a tener una frecuencia más baja que 'posverdad', se documenta desde el año 2000, con una tendencia al alza sostenida en el tiempo desde 2014.

La frecuencia de aparición de las voces es, pues, un criterio complejo, tal y como pone de manifiesto la bibliografía especializada, ya que no basta determinar la frecuencia absoluta de una voz sino que hay que documentar su estabilización, muestra de que el neologismo se ha implantado en el uso. Asimismo, la frecuencia resulta un criterio de difícil aplicación debido a las limitaciones inherentes de los corpus.

Por eso, la práctica lexicográfica aplica una conjunción de criterios ponderados de algún modo para decidir sobre la incorporación de nuevas voces en una obra lexicográfica. En el caso del diccionario académico, los criterios empleados, más que actuar como filtros de exclusión, parecen tomarse como méritos a favor de la inclusión de determinados vocablos. En este sentido, aquellos que parecen tener mayor peso en la decisión son los criterios relacionados con la coherencia interna de la obra: por un lado, se observa que numerosas de las voces nuevas que se incorporan completan series derivativas; por otro, se enriquece el léxico de áreas temáticas presentes en el diccionario, que completan así su cobertura, a pesar de que en algunos casos se trate de voces muy especializadas, característica que aparentemente tendría que ponderarse como un aspecto negativo. Sin embargo, la actualización del léxico especializado se convierte en un criterio complejo, ya que la voluntad de cubrir adecuadamente un área de especialidad puede imponerse a la frecuencia. En este segundo caso, junto con la necesidad de coherencia en la representación de vocabulario temático se tiene en consideración la necesidad denominativa. En esta misma dirección de la coherencia interna parecen apuntar los criterios pragmáticos, pues el diccionario recoge en su última edición nuevas voces de registro coloquial, registro que tradicionalmente ha tenido una representación pobre en esta obra y que de este modo se enriquece.

Lo mismo que ocurre en relación con la frecuencia parece observarse, en el caso del diccionario académico, en relación con los criterios documentales, puesto que gran parte del vocabulario que se añade a su última edición no está recogido en diccionarios de referencia, ni del español ni de otras lenguas. Frente a ello, en cambio, el diccionario parece aplicar un filtro de buena formación, pues ninguna de las nuevas voces incorporadas presenta anomalías formales.

Los criterios aplicados por la Academia, deducibles a partir de las decisiones que ha tomado en su última edición, no son idénticos a los que se aplican en otros diccionarios, como hemos visto con el análisis de los 50 neologismos más frecuentes: 
el DLE se sitúa en un extremo de menor apertura a los neologismos mientras que el NEOMM, inmediatamente seguido por el DUE4, se sitúa en el otro extremo. Ello apunta a que los criterios de diccionariabilidad son dependientes del tipo de diccionario, de modo que en función de las características de cada obra lexicográfica no solo unos u otros criterios tendrán mayor o menor peso, sino que actuarán como filtros de exclusión o serán tomados como méritos para la inclusión.

\section{CONCLUSIONES}

Los criterios que deben seguir las obras lexicográficas para sancionar la inclusión de voces neológicas han sido objeto de discusión y debate en la bibliografía especializada tanto en lexicografía como en neología. Especialmente en la última década, se han publicado numerosos trabajos que analizan la cuestión desde puntos de vista diversos y proponen criterios cada vez más refinados para la valoración de la diccionarización de los neologismos. En su conjunto, los estudios realizados abogan por la toma en consideración de un conjunto de rasgos de distinta naturaleza, que deben ser ponderados adecuadamente para su aplicación. Las sucesivas propuestas realizadas añaden rasgos específicos a la descripción, plantean pesos relativos distintos entre los rasgos tenidos en cuenta en la ponderación o refinan su caracterización; todas ellas, sin embargo, comparten las grandes líneas en el establecimiento de los criterios para la diccionarización de voces neológicas. En concreto, coinciden en conceder un papel preponderante a los factores relacionados con la frecuencia de uso, que en muchas ocasiones es tomada con un filtro sine qua non, así como en combinar este criterio con factores relativos a las cuestiones formales y semánticas — no siempre desligadas unas de las otras - y a cuestiones documentales, esto es, inclusión en otras fuentes lexicográficas de referencia.

En este estado de cosas, el análisis de la práctica lexicográfica en relación con la entrada de voces nuevas se podría entender, consiguientemente, como un ejercicio de crítica lexicográfica, en la medida en que el análisis permite contrastar la aplicación, en una determinada obra, de los criterios establecidos en la bibliografía. Se pueden entender también, sin embargo, como un ejercicio de crítica metalexicográfica, en la medida en que la identificación de los criterios aplicados en los diccionarios permite contrastar la validez de los criterios propuestos en los trabajos especializados. En este sentido, el trabajo que aquí se ha presentado invita a una reflexión sobre el hecho mismo de pretender establecer criterios para la inclusión de neología en 'el' diccionario. La diccionariabilidad, parece, no es un rasgo absoluto de las voces neológicas, sino relativo al tipo de diccionario. Así, el criterio de la coherencia interna de la obra se superpone a todos los demás e informa la toma de decisiones, de modo que, por ejemplo, los criterios de frecuencia pueden ser concebidos como un filtro que deben cumplir todas las voces recogidas en una determinada obra lexicográfica, pero pueden ser criterios que tengan una importancia muy menor en otras obras. 
En el caso del diccionario académico aquí analizado, por ejemplo, el análisis de la neología recogida en la última edición permite inferir la aplicación de ciertos criterios implícitos — con algunas incoherencias, ciertamente, pero que no llegan a impedir su identificación_- En primer lugar, se aplica un criterio fundamental de corrección formal, pues ninguna de las voces incluidas presenta anomalías en su formación. En segundo lugar, se observa una voluntad de completar determinadas parcelas del léxico, ya sea con la compleción de series derivativas o con la incorporación de vocabulario de áreas temáticas determinadas. En esta labor, el papel concedido a la frecuencia no es central, puesto que determinadas voces son repertoriadas en el diccionario si cumplen con una función denominativa considerada necesaria en esa parcela, sino que el criterio fundamental es la coherencia interna de la obra. También tiene que ver con la coherencia interna la incorporación de léxico dialectal o de registro coloquial; en este caso, sin embargo, la frecuencia sí parece ser un filtro necesario, puesto que el diccionario académico solo incluye voces coloquiales o dialectales de frecuencia alta.

Actualizar un diccionario es, en definitiva, una tarea que se debe emprender combinando criterios de índole diversa para intentar reflejar del mejor modo posible la consolidación de un léxico que cambia incesantemente desde una edición del diccionario a la siguiente, con el fin de dar así adecuada cuenta de la vitalidad de la lengua y de los usos lingüísticos de sus hablantes en cada momento.

\section{REFERENCIAS BIBLIOGRÁFICAS}

Adelstein, A. \& Freixa, J. (2013). Criterios para la actualización lexicográfica a partir de datos de observatorios de neología. Comunicación presentada en el Congreso Internacional El diccionario: Neología, lenguaje de especialidad, computación, 28-30 de octubre. México: Ciudad de México [en línea]. Disponible en: https:/ / repositori.upf.edu/handle/10230/34891

Apresjan, J. D. (1974). Regular polysemy. Linguistics, 12(142), 5-32.

Auger, P. \& Rousseau, L. (1984). Metodología de la investigación terminológica. Málaga: Universidad de Málaga.

Barnhart, D. K. (1985). Prizes and pitfalls of computerized searching for new words for dictionaries. Dictionaries, 7, 253-260.

Base de dades de l'Observatori de Neologia (BOBNEO) (1989). Barcelona: Institut de Lingüística Aplicada -Universitat Pompeu Fabra [en línea]. Disponible en: http://obneo. iula.upf.edu/bobneo/index.php

Beniers, E. (1990). La derivación en la gramática y en el diccionario. Estudios de Lingüística Aplicada, 11, 102-115. 
Bernal, E. (2003). Morfología y diccionario. Lagunas léxicas y lagunas lexicográficas en los sustantivos deverbales del sufijo -itzar del catalán. En C. Lacobini \& A. Thornton (Eds.), Scritti di morfologia in onore di Sergio Scalise in occasione del suo 68 compleanno (pp. 13-28). Cesena: Caissa Italia.

Bernal, E. (2015). Ser o no ser: Els neologismes i la percepció dels parlants, entre la normalitat i la raresa. En C. Sinner \& K. Wieland (Eds.), Norma, ús $i$ actituds lingüistiques. El paper del català en la vida quotidiana (pp. 61-73). Leipzig: Leipziger Universitätsverlag.

Borrego Nieto, J. (1999). El español de Castilla y León: ¿Modelo lingüístico o complejo dialectal? En A. Álvarez Tejedor (Coord.), La lengua española patrimonio de todos (pp. 13-37). Burgos: Caja de Burgos.

Boulanger, M. (1997). What makes a coinage successful?: The factors influencing the adoption of English new words. Tesis doctoral, University of Georgia, Athens, Estados Unidos.

Cook, C. P. (2010). Exploiting linguistic knowledge to infer properties of neologisms. Tesis doctoral, University of Toronto, Toronto, Canadá.

Estopà, R. (2015). Sobre neologismos y neologicidad: Reflexiones teóricas con repercusiones metodológicas. En I. M. Alves \& E. Simões Pereira (Eds.), Neologia das Linguas Românicas (pp. 111-150). São Paulo: CAPES; Humanitas.

Freixa, J. (2010). Paraules amb rareses. Terminalia, 1, 7-16.

Freixa, J. (2016). Reagrupamiento lexicográfico de neologismos frecuentes. En E. Bernal \& S. Torner (Eds.), Relaciones morfológicas y diccionario (pp. 95-113). A Coruña: Universidade da Coruña.

Freixa, J. (en prensa). Criteris per a la diccionarització de neologismes. En B. Meisnitzer \& J. Martines (Eds.), Canvi lingüistic, estandardització $i$ identitat en català. Leipzig: Leipziger Universitätsverlag.

García de Diego, V. (1950). El castellano como complejo dialectal y sus dialectos internos. Revista de Filología Española, 34, 107-124.

Ishikawa, S. (2006). When a word enters the dictionary: A data-based analysis of neologism. En JACET Society of English Lexicography (Ed.), English Lexicography in Japan (pp. 39-52). Bunkyo-ku: Taishukan.

Metcalf, A. (2002). Predicting new words. Boston: Houghton Mifflin Company.

Observatori de Neologia (2004). Metodologia del treball en neologia: Criteris, materials $i$ processos. Barcelona: Institut de Lingüística Aplicada - Universitat Pompeu Fabra. 
O’Donovan, R. \& O’Neill, M. (2008). A systematic approach to the selection of neologisms for inclusion in a large monolingual dictionary. En E. Bernal \& J. DeCesaris (Eds.), Proceedings of the XIII EURALEX International Congress (Barcelona, 15-19 July 2008) (pp. 571-579). Barcelona: Institut de Lingüística Aplicada - Universitat Pompeu Fabra.

Oxford English Dictionary. Oxford: Oxford University Press (2017) [en línea]. Disponible en: http://www.oed.com.

Real Academia Española \& Asociación de Academias de la Lengua Española (2009). Nueva gramática de la lengua española. Madrid: Espasa.

Real Academia Española (2014). Corpus del Español del Siglo XXI (versión 0.83) [en línea]. Disponible en: http://web.frl.es/CORPES/view/inicioExterno.view (fecha de consulta: 10 de junio de 2018).

Real Academia Española \& Asociación de Academias de la Lengua Española (2014). Diccionario de la lengua española (23. a ed.). Madrid: Espasa.

Real Academia Española (2015). Corpus de Referencia del Español Actual (versión anotada 0.1) [en línea]. Disponible en: http://web.frl.es/CREA/view/inicio Externo.view (fecha de consulta: 12 de junio de 2018).

Sánchez Manzanares, C. (2013). Valor neológico y criterios lexicográficos para la sanción y censura de neologismos en el diccionario general. Sintagma, 25, 111 125.

Sanmartín, J. (2016). Sobre neología y contextos de uso: Análisis pragmalingüístico de lo ecológico y de lo sostenible en normativas y páginas web de promoción turística. Ibérica, 31, 175-198.

Torner, S. (2013). Suffix -mente adverbs in DAELE, a Spanish learner's dictionnary. International Journal of Lexicography, 26(4), 469-497. 


\section{NOTAS}

${ }^{1}$ Este trabajo forma parte del proyecto de investigación La medición de la neologicidad y la diccionariabilidad de los neologismos del español (Neómetro), ref. FFI2016-79129-P (AEI/FEDER, UE), financiado por el Ministerio de Economía y Competitividad.

${ }^{2}$ En Freixa (en prensa), por ejemplo, encontramos la aplicación y cuantificación de criterios para establecer la diccionariabilidad de 2.500 neologismos con el objetivo de actualizar el diccionario normativo catalán.

${ }^{3}$ El Corpus del Español del Siglo XXI (CORPES XXI) cuenta en su versión 0.91 (diciembre de 2018) con más de 286 millones de formas, procedentes de textos, orales y escritos, tanto de España como de América, de 2001 en adelante.

${ }^{4}$ Para el recuento, no incluimos ni post ni 'tableta', por la dificultad de contabilizar por separado los usos neológicos: en el primer caso, existen frecuentes usos del prefijo escrito como palabra independiente, mientras que en el segundo aparecen numerosas ocurrencias referidas a tablas pequeñas $\mathrm{y}$, especialmente, a las de chocolate. La forma no adaptada al español tablet aparece documentada 790 ocasiones en 357 documentos.

${ }^{5}$ El Corpus de Referencia del Español Actual (CREA) cuenta en su última versión (3.2, de junio de 2008) con 160 millones de formas, procedentes de textos escritos y orales producidos entre 1975 y 2004 en todos los países de habla hispana.

${ }^{6}$ Cabe decir que ni CORPES XXI ni BOBNEO registran ningún ejemplo del verbo, si bien es fácilmente documentable en la red: “Claves para 'posturear' mejor" (El Huffington Post, 21/03/2013); “[...] aquí tenéis unos trucos que podéis sumar a los que ya os dimos sobre 'Términos para 'posturear' con una copa de vino' para parecer un experto" (La Rioja.com, 15/03/2017).

${ }^{7}$ De nuevo, se trata de palabras de frecuencia muy baja: minicasino solo presenta 2 ocurrencias en BOBNEO y niguatoso, - sa no aparece ni en BOBNEO ni en CORPES XXI. 